\title{
ADSORCIÓN DEL COLORANTE AZUL DIRECTO 2 SOBRE BORRA DE CAFE EN UNA COLUMNA DE LECHO FIJO A ESCALA DE LABORATORIO
}

\section{ADSORPTION OF DIRECT BLUE 2 DYES OVER A COFFEE GROUNDS IN A FIXED-BED COLUMN AT LABORATORY SCALE}

\author{
Grey Cecilia Castellar-Ortega ${ }^{1}$, María Mercedes Cely-Bautista ${ }^{2}$, Beatriz María Cardozo-Arrieta ${ }^{3}$, \\ Edgardo Ramón Angulo-Mercado ${ }^{4}$, Martín Elías Plaza-Vega ${ }^{5}$
}

${ }^{1}$ Ingeniera Química, M.Sc. Ciencias Química, cPh.D. Ciencias Química. Universidad Autónoma del Caribe, Docente, Grupo de Interdisciplinario de Investigación en Energía y Medio Ambiente. Calle 90 No. 46-112, Barranquilla, Colombia, e-mail: grey.castellar@uac.edu.co, (iD https://orcid.org/0000-0001-7711-5912; ${ }^{2}$ Ingeniera Metalúrgica, M.Sc. Ingeniería Mecánica, Ph.D. Ciencia e Ingeniería de Materiales. Universidad Autónoma del Caribe, Docente Investigador, Grupo de Investigación en Materiales Procesos y Tecnologías de Fabricación. Calle 90 No. 46-112, Barranquilla, Colombia, e-mail: mmcelly@yahoo. es, (Dhttp://orcid.org/0000-0003-2980-8807; ${ }^{3}$ Ingeniera de Materiales, M.Sc. Ciencias Ambientales. Universidad Autónoma del Caribe, Docente, Grupo de Investigación en Materiales Procesos y Tecnologías de Fabricación. Calle 90 No. 46-112, Barranquilla, Colombia, e-mail: bcardozo@uac.edu.co, (iDhttps://orcid.org/0000-0002-0112-3622; ${ }^{4}$ Licenciado en Química, M.Sc. Ciencias Química. Universidad del Atlántico, Grupo de Investigación en Biotecnología de Microalgas, Fisicoquímica Aplicada y Estudios ambientales, Docente. km 7 antigua vía Puerto Colombia, Atlántico-Colombia, e-mail: edgangulo20@ gmail.com, (iDhttps://orcid.org/0000-0003-4884-5099; ${ }^{5}$ Ingeniero de Materiales. CSPTubo360 Ltda, Supervisor de Producción Tratamientos Térmicos. Km 7 variante Malambo-Caracolí, Atlántico-Colombia, e-mail: martin.plaza93@gmail.com, (iDhttps://orcid.org/0000-0003-3535-5744

\author{
Rev. U.D.C.A Act. \& Div. Cient. 21(2): 531-541, Julio-Diciembre, 2018 \\ https://doi.org/10.31910/rudca.v21.n2.2018.971
}

Artículo de acceso abierto publicado por Revista U.D.C.A Actualidad \& Divulgación Científica bajo una licencia Creative Commons CC BY-NC 4.0

\section{RESUMEN}

La contaminación del agua con colorantes sintéticos, resultado de las actividades industriales, es un problema latente en la sociedad. Su presencia, aun en concentraciones muy bajas, influye drásticamente en los procesos de fotosíntesis, porque impide la penetración de la luz, afectando la vida acuática e, incluso, la salud humana. La adsorción con carbón activado es una de las técnicas más empleadas para remover el color de los efluentes, pero sus altos costos han dirigido la atención de los investigadores hacia el estudio de materiales adsorbentes provenientes, principalmente, de la agroindustria. Este artículo presenta un estudio sobre la remoción del colorante azul directo 2 en disolución acuosa sobre borra de café sin tratar y modificada a $30 \pm 1^{\circ} \mathrm{C}$, en una columna empacada. Se realizó la caracterización fisicoquímica de los adsorbentes, que incluye la evaluación de las propiedades de textura, mediante isotermas de adsorción, con $\mathrm{N}_{2}$ a 77K, la identificación y cuantificación de grupos funcionales orgánicos, con FTIR y el método de Boemh. El estudio en columna evaluó el efecto de la altura del lecho del adsorbente $(Z=3$ y $6 \mathrm{~cm})$, el flujo volumétrico $\left(Q_{\mathrm{v}}=2\right.$ y $\left.4 \mathrm{~cm}^{3} \mathrm{~min}^{-1}\right)$ y la concentración inicial $\left(C_{o}=8\right.$ y $\left.14 \mathrm{mgdm}^{-3}\right)$, sobre el tiempo de ruptura y la capacidad de adsorción. Los datos experimentales de las curvas de ruptura se ajustaron al modelo BDST (Bed Depth Service Time). Los resultados muestran que el rendimiento de la columna mejora con el incremento de $\mathrm{Z}$ y la disminución de Qv y Co, siendo la concentración inicial, el factor con mayor significancia.

Palabras clave: remoción, contaminantes, tratamiento de aguas residuales, adsorción.

\section{ABSTRACT}

Water bodies contaminated with synthetic dyes from industrial activities is a latent problem in our society. Even in low concentration it influences drastically in photosynthesis process 
because it blocks sun rays affecting both life in water and human health as well. Adsorption with activated carbon is one of the most used techniques to remove dyes from effluents, but high costs have lead researchers to examine adsorbents materials coming from agroindustry. This article introduces a research about direct blue 2 removal in water solution over coffee grounds untreated and modified at $30 \pm 1^{\circ} \mathrm{C}$ in a packed column. A physicochemical characterization was carried out in adsorbents that involves the test of texture properties using isotherms of adsorption with $\mathrm{N}_{2}$ at $77 \mathrm{~K}$, the identification and quantification of organic functional groups with FTIR and the Boemh method. The study in column, tested the effect of the height of the bed of the adsorbent $(Z=3 y$ $6 \mathrm{~cm})$, the volumetric flow $\left(Q_{v}=2\right.$ y $\left.4 \mathrm{~cm}^{3} \mathrm{~min}^{-1}\right)$ and the initial concentration $\left(\mathrm{C}_{\mathrm{o}}=8 \mathrm{y} 14 \mathrm{mgdm}^{-3}\right)$ at the breakthrough time and the capacity of adsorption. The experimental data of breakthrough curves were fitted to BDST (Bed Depth Service Time) models. The results show that performance in the column improves as $\mathrm{Z}$ increases and $\mathrm{Q}_{\mathrm{v}} \mathrm{y}$ Co decreases, being the initial concentration the factor with main significance.

Keywords: removal, pollutants, wastewater treatment, adsorption.

\section{INTRODUCCIÓN}

La contaminación del ambiente por colorantes constituye un problema crítico en el mundo y, es por ello, que es importante la búsqueda de alternativas para su solución. Debido a su compleja estructura química y origen sintético, los colorantes son resistentes a la decoloración por exposición a la luz, al calor, al agua, a los detergentes y a muchos productos químicos (Kyzas et al. 2012; Jung et al. 2016). En el caso específico de esta investigación, el colorante azul directo 2 (DB2), se utiliza, principalmente, para el teñido de pantalones tipo jean, se caracteriza por ser muy soluble en agua y difícil de degradar, bajo condiciones ambientales normales.

Hay varios métodos reportados para la eliminación de colorantes en aguas residuales y estas tecnologías se pueden dividir en tres importantes categorías: los métodos químicos, biológicos y físicos (Castellar et al. 2013); sin embargo, todos ellos tienen sus ventajas y desventajas (Adegoke \& Bello, 2015), pero, principalmente, por razones de costos y problemas de disposición que conducen a la generación de lodos o la formación de subproductos, muchos de estos métodos convencionales para el tratamiento del color, tales como la coagulación, la oxidación química, los procesos de separación por membrana, electroquímicos y degradación microbial aerobia y anaerobia, no se aplican ampliamente, a gran escala, en la industria de textiles y papel (Devi et al. 2008). En este sentido, entre todos estos métodos, la adsorción ha ganado importancia, debido a su eficacia en la eliminación de varios contaminantes y facilidad de aplicación (Elsherbiny,
2013; Mohammed et al. 2016). Además, los procesos de adsorción permiten la regeneración del adsorbente y la recuperación de contaminantes en soluciones concentradas, a través de ciclos de adsorción-desorción, en muchos casos, permitiendo la reutilización en el proceso, que se puede convertir en una ventaja económica (Dias et al. 2017).

La literatura científica ha reportado potenciales adsorbentes no convencionales para la remoción de colorantes, entre los que se incluye, bioadsorbentes naturales, materiales industriales y agroindustriales (Kyzas et al. 2012; Pachathu et al. 2016). El café es uno de los productos agrícolas cultivado en gran extensión en el mundo. Es utilizado, principalmente, para la preparación del café como bebida, a partir de granos molidos, siendo la borra de café el principal desecho de la industria y comercio dedicados a esta actividad (Urribarrí et al. 2014). Para el 2010, según reportes de la Organización Internacional del Café, la producción mundial de granos de café sobrepasó los 8 millones de toneladas, lo cual, indica que se generan grandes cantidades de borra de café (Urribarrí et al. 2014), cuya disposición final, en gran parte, son los rellenos sanitarios, pero que bien se pueden aprovechar en la preparación de adsorbentes.

Los estudios de adsorción en disolución, mediante experimentos por lotes, proporcionan una medida de la eficacia de la adsorción en la eliminación de adsorbibles específicos, así como la capacidad máxima de adsorción (Saleh \& Al-Absi, 2017). Tales experimentos consisten en colocar una cantidad determinada de adsorbente en contacto con la disolución que contiene la sustancia que se va a remover, manteniéndolo en suspensión, mediante agitación el tiempo necesario, para alcanzar el equilibrio.

Los estudios de adsorción en una columna de lecho fijo, a diferencia de los experimentos por lotes, no funcionan bajo condiciones de equilibrio, debido a que continuamente ingresa a la columna una disolución de alimentación, donde se establece un proceso de transferencia de masa permanente entre una fase móvil, que contiene la sustancia que se va a remover y la fase sólida del lecho con material adsorbente. Los procesos industriales, generalmente, se trabajan bajo condiciones continuas, por lo tanto, este tipo de estudios proporciona la aplicación más práctica en el tratamiento de aguas residuales (Valencia et al. 2013).

Esta investigación estudió el efecto de la concentración de alimentación ( $\left.\mathrm{C}_{\mathrm{o}}\right)$, el flujo volumétrico $\left(\mathrm{Q}_{\mathrm{v}}\right)$ y la altura del lecho (Z), sobre la capacidad de adsorción en una columna empacada con borra de café sin tratar y modificada, manteniendo la temperatura y $\mathrm{pH}$ inicial constantes, en la remoción del colorante DB2. De igual manera, los resultados de los tiempos de ruptura se ajustaron al modelo del tiempo de servicio de la altura de un lecho (BDST, de sus iniciales en idioma inglés). Los 
valores de las variables de proceso corresponden a un diseño experimental a escala de laboratorio; sin embargo, existen esfuerzos por parte del grupo de trabajo en probar otros valores a escala piloto y su influencia bajo condiciones dinámicas.

\section{MATERIALES Y MÉTODOS}

Preparación de la borra de café tratada: El método de preparación del café consistió en llenar con $15 \mathrm{~L}$ de agua del grifo, aproximadamente, el depósito de una cafetera de goteo, marca Black \& Decker; luego, se agregó $100 \mathrm{~g}$ de café tostado y molido, de una marca comercial, suministrado por el Departamento de Ciencias Básicas de la Universidad Autónoma del Caribe. La bebida caliente, producto de la extracción, se recogió en una jarra de vidrio y el residuo del café, es lo que se conoce como borra de café.

La borra de café se lavó con agua destilada caliente, para eliminar finos e impurezas (Lafi et al. 2016), hasta lograr un pH constante, de 6,7, medido en un equipo pH HQ11d Portable pH/ORP Meter marca HACH ${ }^{\circledR}$. La borra húmeda se secó en un horno, a $100^{\circ} \mathrm{C}$ por $24 \mathrm{~h}$; luego, se impregnó con ácido fosfórico (Ma et al. 2013), al 20\%m/v, en una proporción de $4 \mathrm{~cm}^{3}$ de solución de $\mathrm{H}_{3} \mathrm{PO}_{4}$, por cada gramo de borra de café seca. Se mantuvo en agitación por $4 \mathrm{~h}$ con ultrasonido (Ma et al. 2013) y, por último, se calentó a $100^{\circ} \mathrm{C}$ durante $1 \mathrm{~h}$, en una mufla en atmósfera de aire. Para eliminar el exceso de ácido fosfórico, se lavó con agua destilada, hasta lograr un $\mathrm{pH}$ constante $(6,6-6,7)$ y se secó nuevamente, a $100^{\circ} \mathrm{C}$ por 24h, en un horno (Jung et al. 2016). Debido a la presencia de material lignocelulósico: celulosa, hemicelulosa y lignina en la borra de café, se espera que el tratamiento con ácido fosfórico modifique su estructura, formando nuevos grupos superficiales funcionalizados (Tehrani et al. 2015), dentro de un esqueleto rígido de carbono, con predominio en estructuras aromáticas (Yakout et al. 2016).

Caracterización de la borra de café sin tratar y modificada: La cantidad de grupos funcionales orgánicos ácidos, tales como ácidos carboxílicos, fenoles y lactonas, se determinaron por titulación con una serie de bases de diferente fortaleza, basado en el método de Boehm (Boehm, 1966; Nunell et al. 2012). Se asume, que el $\mathrm{NaOH}$ neutraliza a los grupos carboxílicos, lactónicos y fenólicos; el $\mathrm{Na}_{2} \mathrm{CO}_{3}$, a los carboxílicos y lactónicos y, el $\mathrm{NaHCO}_{3}$, solo a los carboxílicos (Boehm, 1966; Kyzas et al. 2012). Este procedimiento consistió en colocar por separado $1 \mathrm{~g}$ de las muestras de adsorbente en $100 \mathrm{~cm}^{3}$ de disoluciones de $\mathrm{NaOH}, \mathrm{Na}_{2} \mathrm{CO}_{3}$ y $\mathrm{NaHCO}_{3}$, previamente estandarizadas, por $24 \mathrm{~h}$, a $25^{\circ} \mathrm{C}$, en agitación constante; posteriormente, se filtró, se tomó una alícuota y se le determinó la concentración final, usando ftalato ácido de potasio (FHK), como estándar primario, para el $\mathrm{NaOH}$ y $\mathrm{HCl} \mathrm{0,1M}$, para el $\mathrm{Na}_{2} \mathrm{CO}_{3}$ y $\mathrm{NaHCO}_{3}$. Los espectros de infrarrojo con transformada de Fourier (FTIR), se midieron en el rango entre 4000 a $400 \mathrm{~cm}^{-1}$, a una velocidad de 20 escaneos por minuto, con una resolución de $2 \mathrm{~cm}^{-1}$, en un espectrofotómetro marca SHIMADZU IRAffinity-1, en pastilla de $\mathrm{KBr}$, en una proporción de 1:100, este, se realizó en el Laboratorio de Bioprocesos de la Universidad. El área específica, se determinó a partir de la isoterma de adsorción con nitrógeno, a $77 \mathrm{~K}$ a presiones relativas, entre 0,05 y 0,3, ajustando los resultados al modelo de Brunauer, Emmett y Teller (BET), en un analizador de área superficial y porosidad Micromeritics Gemini VII 3.03.

Estudio en columna: El montaje experimental consistió en un depósito de almacenamiento, que contiene la solución con el colorante, con la ayuda de una bomba peristáltica, que se alimentó a una columna de vidrio de $2 \mathrm{~cm}$ de diámetro interno, empacada con borra de café sin tratar y modificada, según el caso. Para un caudal determinado $\left(2 \mathrm{y} 4 \mathrm{~cm}^{3} \mathrm{~min}^{-1}\right)$, a dos alturas diferentes $(3 \mathrm{y} 6 \mathrm{~cm})$ y dos concentraciones

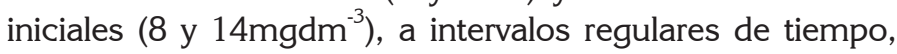
se tomaron alícuotas del fluido a la salida de la columna, para luego ser analizadas por la técnica UV-vis, en un equipo marca Thermo Spectronic Genesys 20, a una longitud de onda de $575 \mathrm{~nm}$, determinada por barrido previo. Todos los ensayos se realizaron por triplicado, a una temperatura de $30^{\circ} \mathrm{C} \pm 1^{\circ} \mathrm{C}$.

El colorante DB2 fue suministrado por una empresa ubicada en Barranquilla-Colombia, dedicada a la fabricación de pantalones tipo jean. El nombre que recibe, según The Color Index, es Direct Blue 2. C.I. 22590, un colorante directo con doble grupo azo, fórmula molecular $\mathrm{C}_{32} \mathrm{H}_{21} \mathrm{~N}_{6} \mathrm{Na}_{3} \mathrm{O}_{11} \mathrm{~S}_{3} \mathrm{y}$ masa molar molecular de 830,71 $\mathrm{gmol}^{-1}$.

Diseño experimental: Se utilizó un diseño experimental factorial $2^{3}$ con dos repeticiones y valores de nivel bajo y alto, para la borra del café sin tratar y modificada. Para este estudio, el factor A, se refiere a la concentración inicial del colorante $(8 \mathrm{y}$ $\left.14 \mathrm{mgdm}^{-3}\right)$, el factor $\mathrm{B}$, a la altura del lecho $(3 \mathrm{y} 6 \mathrm{~cm})$ y factor C, al flujo volumétrico $\left(2\right.$ y $\left.4 \mathrm{~cm}^{3} \mathrm{~min}^{-1}\right)$ y la variable respuesta es la capacidad de adsorción del colorante $\left(\mathrm{mgg}^{-1}\right)$. El efecto de los factores y de las interacciones, se muestra bajo un Análisis de varianza. Los resultados fueron analizados con el software estadístico STATGRAPHICS Centurión XV ® 2006.

\section{RESULTADOS Y DISCUSIÓN}

Caracterización de la borra de café sin tratar y modificada: Los granos secos del café almendra están constituidos, principalmente, por sustancias orgánicas, tales como los carbohidratos, lípidos, proteínas, alcaloides, ácidos carboxílicos y fenólicos y por compuestos volátiles, que dan el aroma (Puerta, 2011). Una estimación de la presencia de compuestos orgánicos ácidos en la borra de café, aun después de 
haber pasado por un riguroso proceso de lavado, secado y tratamiento químico, se muestra en la tabla 1. La presencia de ácidos carboxílicos y fenoles en la borra del café sin tratar y modificada, se debe a los ácidos clorogénicos. Son los más abundantes en la almendra del café, principalmente, el ácido quínico, el ácido cinámico, el ácido sinápico, los ácidos cumáricos, el ácido cafeico y el ácido cafeoilquínico, que es el que más aporta a la acidez. Después de los clorogénicos, los ácidos más abundantes son los carboxílicos alifáticos, como el cítrico, el acético y el málico (Puerta, 2011).
En el caso de la presencia de lactonas es importante mencionar que, en la torrefacción del grano de café, se presentan diferentes reacciones de los ácidos clorogénicos y, en consecuencia, se producen varios compuestos químicos en el grano tostado, que dependen del grado de tostación utilizado (Gutiérrez, 2002). En este proceso, los ácidos clorogénicos se isomerizan, se unen a las melanoidinas, se hidrolizan y forman las quinolactonas (Puerta, 2011). Dos lactonas que han sido identificadas en el café tostado son la 3-cafeoíl y 4-cafeoíl lactona del ácido quínico. Después del tratamiento

Tabla 1. Cuantificación de los grupos funcionales superficiales orgánicos de la borra de café sin tratar y modificada.

\begin{tabular}{|c|c|c|c|c|c|}
\hline Adsorbente & $\begin{array}{c}\text { Acidez total } \\
\left(\text { meqg }^{-1}\right)\end{array}$ & $\begin{array}{c}\text { Ácidos carboxílicos } \\
\left(\text { meqg }^{-1}\right)\end{array}$ & $\begin{array}{c}\text { Lactonas } \\
\left(\text { meqg }^{-1}\right)\end{array}$ & $\begin{array}{c}\text { Fenoles } \\
\left(\text { meqg }^{-1}\right)\end{array}$ & $\begin{array}{c}\text { Área específica } \\
\left(\mathbf{m}^{2} \mathbf{g}^{-1}\right)\end{array}$ \\
\hline Borra de café & 2,0 & 0,5 & 0,5 & 1,0 & 0,424 \\
\hline Borra de café modificada & 1,5 & 1,0 & 0,0 & 0,5 & 0,554 \\
\hline
\end{tabular}

con ácido fosfórico de la borra del café, se observa que las lactonas se hidrolizan por completo, formando nuevamente ácidos carboxílicos fenólicos, aumentando la cantidad de estos en la borra modificada.

El espectro infrarrojo de la borra de café sin tratar y modificada, se muestra en la figura 1. La vibración de estiramiento del enlace $\mathrm{OH}$ aparece alrededor de los $3450 \mathrm{~cm}^{-1}$, es una absorción intensa y amplia (entre $3600-3200 \mathrm{~cm}^{-1}$ ); se sugiere que corresponde a ácidos carboxílicos, alcoholes, fenoles y, posiblemente, a la presencia de enlaces de hidrógeno, formados durante el tratamiento químico o adquiridos durante los ensayos experimentales. Las bandas observadas entre $2910 \mathrm{~cm}^{-1}$ y $2850 \mathrm{~cm}^{-1}$, se puede deber a estiramientos y deformaciones del grupo $\mathrm{C}-\mathrm{H}$, presente en cadenas poliméricas, con enlaces simples (Albis et al. 2017), tipo celulosa, hemicelulosa y lignina (El Messaoudi et al. 2016). La banda entre 2100 y $2000 \mathrm{~cm}^{-1}$ que aparece en la borra de café puede corresponder a estiramientos de enlace tipo nitrilo, $\mathrm{C} \equiv \mathrm{N}$, pero que, luego del tratamiento químico, ya no se observa en la borra modificada. Por la diversidad de grupos funcionales presentes en los materiales estudiados es posible que las bandas en $1735 \mathrm{~cm}^{-1}$ y $1600 \mathrm{~cm}^{-1}$ correspondan al estiramiento del enlace $\mathrm{C}=\mathrm{O}$, característica de los ácidos carboxílicos y compuestos aromáticos, respectivamente. La banda cerca de $1080 \mathrm{~cm}^{-1}$, se puede deber a la presencia de ésteres de ácido fosfórico $\mathrm{P}^{+}-\mathrm{O}^{-}$y a vibraciones simétricas de cadenas de polifosfatos P-O-P (Myglovets et al. 2014) y, por último, la banda

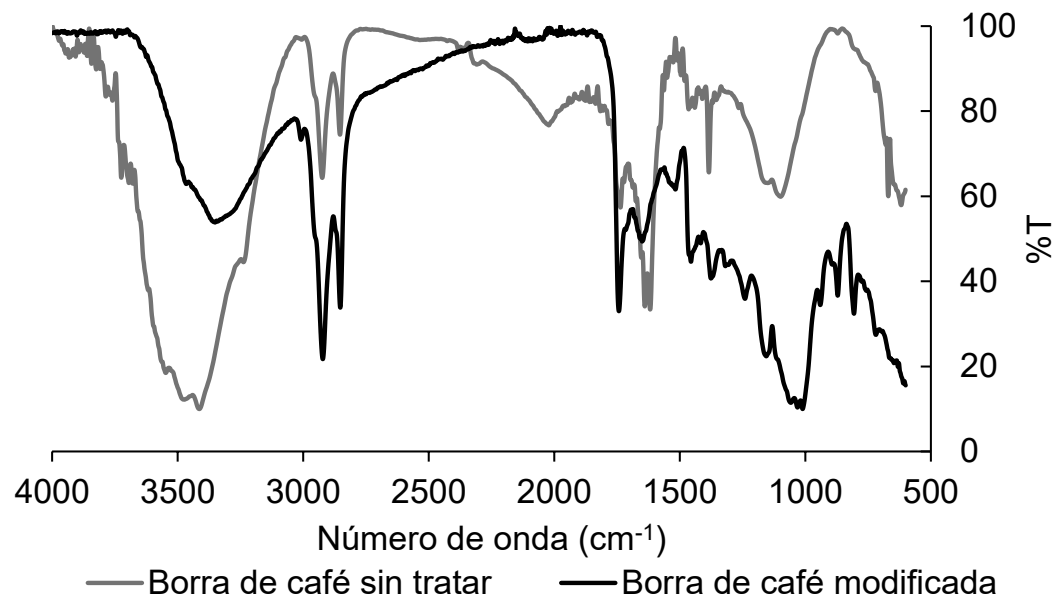

Figura 1. Espectro de infrarrojo de la borra de café sin tratar y modificada. 
de absorción a $1100 \mathrm{~cm}^{-1}$, se atribuye a vibraciones de estiramiento C-O-C en ésteres aromáticos (Fernández et al. 2014). Las bandas de ambos espectros en la región de la huella dactilar no muestran diferencias significativas.

Las bajas áreas superficiales, tanto de la borra de café sin tratar y modificada registradas en la tabla 1, muestran que solo la impregnación con ácido fosfórico no es suficiente para despolimerizar la celulosa, la hemicelulosa y la lignina, presentes en su estructura. Es necesario, además, carbonizarlas, de manera que formen unidades más pequeñas, ricas en carbono, que conducen a un aumento de sus propiedades de textura (Colpas et al. 2015), pero en virtud de explicar las propiedades de remoción exhibidas por los materiales adsorbentes empleados, todo parece indicar que la química de superficie desempeña un papel fundamental, pues la presencia de heteroátomos, principalmente oxígeno, en los diferentes grupos funcionales orgánicos, son los responsables de las propiedades adsorbentes (Nazari et al. 2016).

Estudio de adsorción en columna: La columna empacada con adsorbente se puso en contacto con una disolución acuosa del colorante DB2. Inicialmente, la parte inferior del adsorbente remueve rápidamente el colorante, de manera que la disolución, al salir de la columna, está prácticamente libre de color. Como el volumen de líquido que atraviesa la columna aumenta, la zona de transferencia de masa comienza a desplazarse; la concentración del colorante a la salida, aumenta con el tiempo. Se denomina punto de ruptura al máximo valor en la concentración del colorante, que se puede permitir a la salida de la columna y el tiempo emplea- do para alcanzar esta concentración específica, se le llama tiempo de ruptura (tb) y la curva que resulta de graficar la relación, $\mathrm{C} / \mathrm{C}_{0}$, en función del tiempo, se conoce como curva de ruptura. En este estudio, el criterio de tiempo de ruptura, se adoptó como el lapso que transcurre cuando la concentración del DB2 alcanzó el 10\% de la concentración inicial.

La cantidad de colorante removido por gramo de adsorbente en el punto de ruptura, $\mathrm{q}_{\mathrm{b}}$ en $\mathrm{mgg}^{-1}$, se obtuvo a partir de la ecuación 1.

$$
q_{b}=\frac{Q_{v} t_{b} C_{o}}{1000 m}
$$

Donde tb es el tiempo de ruptura en minutos, $\mathrm{C}_{\mathrm{o}}$ es la concentración inicial del colorante en $\mathrm{mgdm}^{-3}$, Qv es el flujo volumétrico en $\mathrm{cm}^{3} \mathrm{~min}^{-1}$, m es la masa de adsorbente y el valor de 1000 corresponde a un factor de conversión. La tabla 2 muestra los resultados obtenidos para cada una de las condiciones evaluadas. Los mejores valores de adsorción, se obtuvieron a una altura de $6 \mathrm{~cm}$, flujo volumétrico de $2 \mathrm{~cm}^{3} \mathrm{~min}^{-1}$ y concentración inicial de $8 \mathrm{mgdm}^{-3}$, para la borra de café sin tratar $\left(0,033 \mathrm{mgg}^{-1}\right)$ y modificada $\left(0,040 \mathrm{mgg}^{-1}\right)$.

Efecto de la altura del lecho: La figura 2 muestra la curva de ruptura para la adsorción del colorante DB2, a diferentes alturas del lecho $(3$ y $6 \mathrm{~cm})$, a un flujo volumétrico de $2 \mathrm{~cm}$ ${ }^{3} \mathrm{~min}^{-1}$ y una concentración inicial de $8 \mathrm{mgdm}^{-3}$. El tiempo de ruptura se incrementa desde 4,4 a 10,0min, para la borra de café y desde 4,6 a 13,2min, para la borra modificada. Un comportamiento similar se registra para otras condiciones

Tabla 2. Capacidad de adsorción en columna empacada con borra de café sin tratar y modificada.

\begin{tabular}{|c|c|c|c|c|c|c|c|c|}
\hline \multicolumn{3}{|c|}{ Variables de proceso } & \multicolumn{3}{|c|}{ Borra de café } & \multicolumn{3}{|c|}{ Borra de café modificada } \\
\hline $\begin{array}{l}\text { Concentración } \\
\text { inicial } \\
\left(\mathrm{mgdm}^{-3}\right)\end{array}$ & $\begin{array}{c}\text { Flujo } \\
\text { Volumétrico } \\
\left(\mathrm{cm}^{3} \min ^{-1}\right)\end{array}$ & $\begin{array}{l}\text { Altura } \\
\text { del } \\
\text { lecho } \\
\text { (cm) }\end{array}$ & $\begin{array}{l}\text { Tiempo de } \\
\text { ruptura, } \\
\quad=0,1 \\
\text { (min) }\end{array}$ & $\begin{array}{c}\text { Masa del } \\
\text { lecho } \\
\text { (g) }\end{array}$ & $\begin{array}{c}\text { Capacidad } \\
\text { de adsorción } \\
\text { de la co- } \\
\text { lumna } \\
\left(\mathrm{mgg}^{-1}\right)\end{array}$ & $\begin{array}{l}\text { Tiempo de } \\
\text { ruptura, } \\
\quad=0,1 \\
\text { (min) }\end{array}$ & $\begin{array}{c}\text { Masa del } \\
\text { lecho } \\
\text { (g) }\end{array}$ & $\begin{array}{c}\text { Capacidad } \\
\text { de adsorción } \\
\text { de la co- } \\
\text { lumna } \\
\left(\mathrm{mgg}^{-1}\right)\end{array}$ \\
\hline 8 & 2 & 3 & 4,4 & 2,97 & 0,024 & 4,6 & 2,92 & 0,027 \\
\hline 8 & 2 & 6 & 10 & 4,80 & 0,033 & 13,2 & 4,86 & 0,040 \\
\hline 8 & 4 & 3 & 1,0 & 2,94 & 0,011 & 1,4 & 3,00 & 0,020 \\
\hline 8 & 4 & 6 & 3,0 & 5,00 & 0,019 & 3,6 & 4,89 & 0,022 \\
\hline 14 & 2 & 3 & 1,5 & 2,98 & 0,014 & 1,8 & 3,01 & 0,019 \\
\hline 14 & 2 & 6 & 2,1 & 4,70 & 0,016 & 2,5 & 4,78 & 0,020 \\
\hline 14 & 4 & 3 & 0,3 & 2,96 & 0,006 & 0,6 & 3,05 & 0,008 \\
\hline 14 & 4 & 6 & 0,5 & 4,90 & 0,009 & 0,9 & 4,78 & 0,012 \\
\hline
\end{tabular}




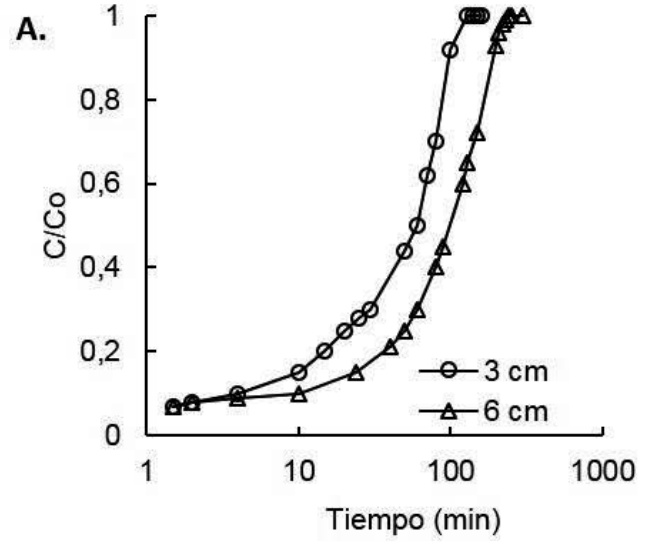

C.

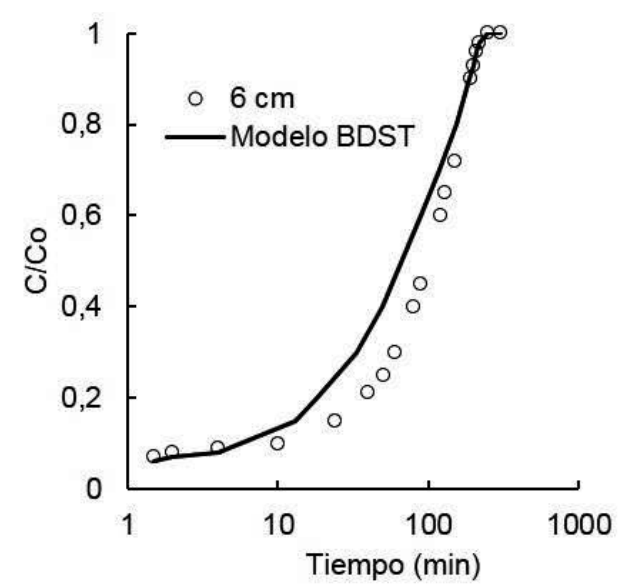

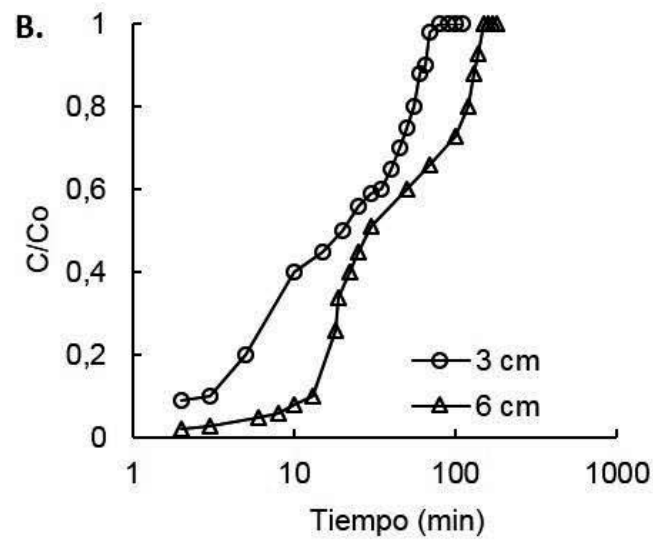

D.

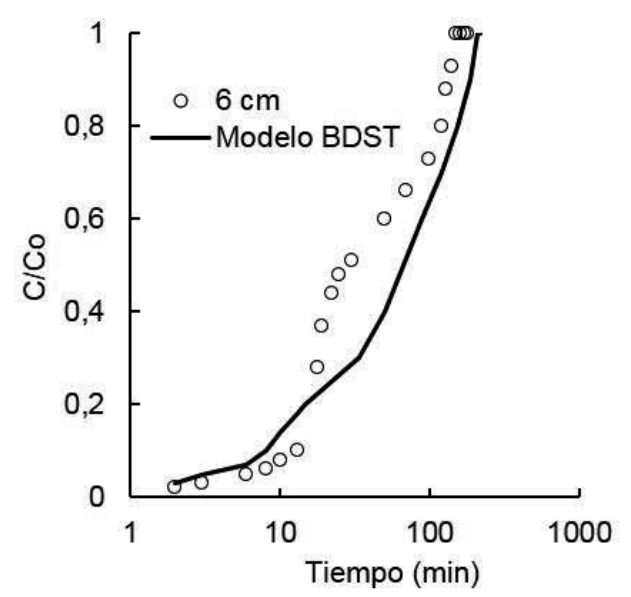

Figura 2. Curvas de ruptura para la adsorción de DB2 en una columna empacada a diferentes alturas, $C_{o}=8 \mathrm{mgdm}^{-3} \mathrm{y} \mathrm{Qv}=$ $2 \mathrm{~cm}^{3} \mathrm{~min}^{-1}$. A) Borra de café; B) Borra de café modificada. Comparación de la curva de ruptura experimental y teórica usando el modelo BDST a $\mathrm{C}_{0}=8 \mathrm{mgdm}^{-3},=0,64 \mathrm{cmmin}^{-1} \mathrm{y}=6 \mathrm{~cm}$; C) Borra de café y D) Borra de café modificada.

de operación evaluadas en este estudio. Cuando el lecho adsorbente aumenta, se dispone de más sitios de adsorción, es decir, de una mayor zona de transferencia de masa (Shanmugam et al. 2016) y, en consecuencia, se mejoran los tiempos de ruptura y la capacidad de adsorción. En estos experimentos, el hecho de que la concentración del colorante DB2 no sea cero después de que el primer caudal haya pasado a través de la columna, indica que la zona de transferencia de masa es de hecho más grande que cada una de estas alturas del lecho. Idealmente, esta situación se evitaría en la práctica al aumentar la profundidad del lecho (Mohammed et al. 2016).

Efecto del flujo volumétrico: De la tabla 2, se observa que el tiempo de ruptura disminuye con el incremento del flujo volumétrico, así cuando el flujo volumétrico aumenta de 2 a $4 \mathrm{~cm}^{3} \mathrm{~min}^{-1}$, el tiempo de ruptura disminuye de 10 a $3 \mathrm{~min}$ y de 13,2 a 3,6min, para la borra de café sin tratar y modificada, respectivamente. Este comportamiento, se debe al tiempo de residencia insuficiente en la columna a flujos volumétricos altos, que no permite que las moléculas del colorante se difundan y se transporten hacia los sitios activos del adsorbente. Igual efecto se produce sobre la capacidad de adsorción, que varía de 0,033 a 0,019 $\mathrm{mgg}^{-1}$, para la borra del café y de 0,040 a $0,022 \mathrm{mgg}^{-1}$, para la borra modificada, cuando

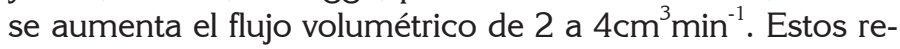
sultados indican que, para lograr una mayor eficiencia de la columna, se deben emplear flujos volumétricos bajos; además se observa que el adsorbente se agota más rápidamente al aumentar el flujo volumétrico, por lo tanto, no se aprovecha toda su capacidad de adsorción (Talat et al. 2018).

Efecto de la concentración inicial: El tiempo de ruptura disminuye de 10,0 a $2,1 \mathrm{~min}$ al incrementar la concentración inicial, desde 8 a $14 \mathrm{mgdm}^{-3}$, para la borra de café sin tratar y de 13,2 a 2,5min, para la borra modificada. Igual comporta- 
miento se presenta para las otras condiciones de operación de la columna, como se muestra en la tabla 2. La disminución del tiempo, en la medida que aumenta la concentración, se puede explicar, considerando que la fuerza motriz del proceso es la diferencia de concentración entre las moléculas del colorante DB2 en el adsorbente y en la disolución, a mayor fuerza motriz, la resistencia a la transferencia de masa disminuye, empleándose menor tiempo en ocupar los sitios activos disponibles del adsorbente.

Otros trabajos muestran un comportamiento similar al aquí reportado, con respecto al efecto de la altura del lecho, la concentración inicial y el flujo volumétrico sobre la capacidad de adsorción de colorantes, en una columna empacada con adsorbentes no convencionales. Dentro las publicaciones, se encuentra el trabajo realizado por Villada et al. (2014), donde remueven el colorante catiónico azul de metileno en una columna de lecho empacado con cascarilla de arroz; el trabajo de El Messaoudi et al. (2016), muestra el uso de cáscara de jujube en la remoción del colorante rojo Congo y, por último, Shanmugam et al. (2016), emplearon un lecho con microalgas Spirulina platensis no viva, para remover el colorante azul reactivo 2 .

Modelo del tiempo de servicio de la altura de un lecho (BDST): Este modelo supone que la difusión intraparticular y la resistencia de masa externa son insignificantes y que la cinética de adsorción está controlada por la reacción superficial entre el soluto en la disolución y el adsorbente, que no ha sido usado (Valencia et al. 2013). Fue propuesto por Hutchins (Hutchins, 1973) y está basado en la ecuación de Bohart y Adams (Bohart et al. 1920). Este establece una relación entre el tiempo de servicio (t) y la altura del lecho (Z), en términos de parámetros de adsorción (Talat et al. 2018). La forma lineal del modelo BDST, se puede escribir como se muestra en la ecuación 2:

$$
\mathrm{t}=\frac{\mathrm{N}_{\mathrm{o}} \mathrm{Z}}{\mathrm{C}_{\mathrm{o}} \mathrm{U}}-\frac{1}{\mathrm{KC}_{\mathrm{o}}} \ln \left(\frac{\mathrm{C}_{\mathrm{o}}}{\mathrm{C}}-1\right)
$$

Donde, $t$ es el tiempo de servicio de la columna en el punto de ruptura (min), es la capacidad dinámica del lecho $\left(\mathrm{mgdm}^{-3}\right)$, es la altura del lecho $(\mathrm{cm}), U$ es la velocidad de flujo lineal $\left(\mathrm{cmmin}^{-1}\right)$, definida como la razón entre el flujo volumétrico Qv $\left(\mathrm{cm}^{3} \mathrm{~min}^{-1}\right)$ y el área de la sección transversal de la columna $\left(\mathrm{cm}^{2}\right)$, Co y C son, respectivamente, la concentración inicial y a un tiempo $\mathrm{t}\left(\mathrm{mgdm}^{-3}\right)$ y, $\mathrm{K}$ es la constante de velocidad de adsorción ( $\mathrm{dm}^{3} \mathrm{~min}^{-1} \mathrm{mg}^{-1}$ ) (Valencia et al. 2013).

La ecuación 2, se puede reescribir en forma de línea recta como sigue:

$$
\mathrm{t}=\mathrm{aZ}-\mathrm{b}
$$

Donde, a y b son la pendiente y la ordenada al origen, respectivamente:

$$
\begin{gathered}
\mathrm{a}=\frac{\mathrm{N}_{\mathrm{o}}}{\mathrm{C}_{\mathrm{o}} \mathrm{U}} \\
\mathrm{b}=\frac{1}{\mathrm{KC}_{\mathrm{o}}} \ln \left(\frac{\mathrm{C}_{\mathrm{o}}}{\mathrm{C}}-1\right)
\end{gathered}
$$

Las figuras 2c y d muestran el ajuste de los datos experimentales al modelo BDST a $\mathrm{C}_{0}=8 \mathrm{mgdm}^{-3}, U=0,64 \mathrm{cmmin}^{-1} \mathrm{y}$ $\mathrm{Z}=6 \mathrm{~cm}$, tanto para la borra de café sin tratar como modificada. Se observa que en el intervalo comprendido entre $0,1<\mathrm{C} / \mathrm{Co}>0,7$ se presentan las mayores desviaciones al modelo, lo cual, indica posibles dificultades difusionales y de transporte, en el proceso de adsorción en columna.

Diseño factorial: Para considerar los efectos de los factores y las interacciones de los efectos, se trabajó con un nivel de confiabilidad de $95 \%$, con un valor de $p<0,05$. La tabla 3 muestra el análisis de varianza para la capacidad de adsorción de los absorbentes; en ella, se prueba la significancia estadística de cada efecto, comparando el cuadrado medio contra un estimado del error experimental. Para el caso de la borra de café cinco efectos tienen un valor -P menor a 0,05, como son la concentración, el flujo, la altura del lecho, la interacción concentración-flujo y la interacción concentración-altura, todos ellos indican que son significativamente diferentes de cero, con un nivel de confianza del 95\%, es decir, que tienen un efecto estadísticamente significativo sobre la capacidad de adsorción. Para el caso de la borra de café modificada, solo los efectos principales tienen un efecto significativo, es decir, la concentración, el flujo y la altura del lecho.

La figura 3 muestra el diagrama de Pareto, para a) la borra de café sin tratar y b) modificada. Este diagrama es una representación gráfica del análisis de varianza; en él, se muestra claramente los factores que tienen un efecto significativo sobre la adsorción del colorante DB2, de tal manera que, cada uno de los factores estimados, se encuentra en orden decreciente de importancia. La línea vertical, se utiliza para juzgar cuáles efectos son estadísticamente significativos; la longitud de cada barra es proporcional al efecto estandarizado, que será el efecto estimado dividido entre su error estándar; cualquier barra que se extienda más allá de la línea, corresponde a efectos que son estadísticamente significativos, con un nivel de confianza del $95 \%$.

De la figura 3, se observa que, en el proceso de adsorción del colorante DB2 sobre la borra de café sin tratar y modificada, la concentración encabeza en orden de importancia y de significancia estadística, como factor predominante en el proceso. Con este resultado, se puede afirmar que la concentración para el presente estudio fue el factor más significativo; por 
A.

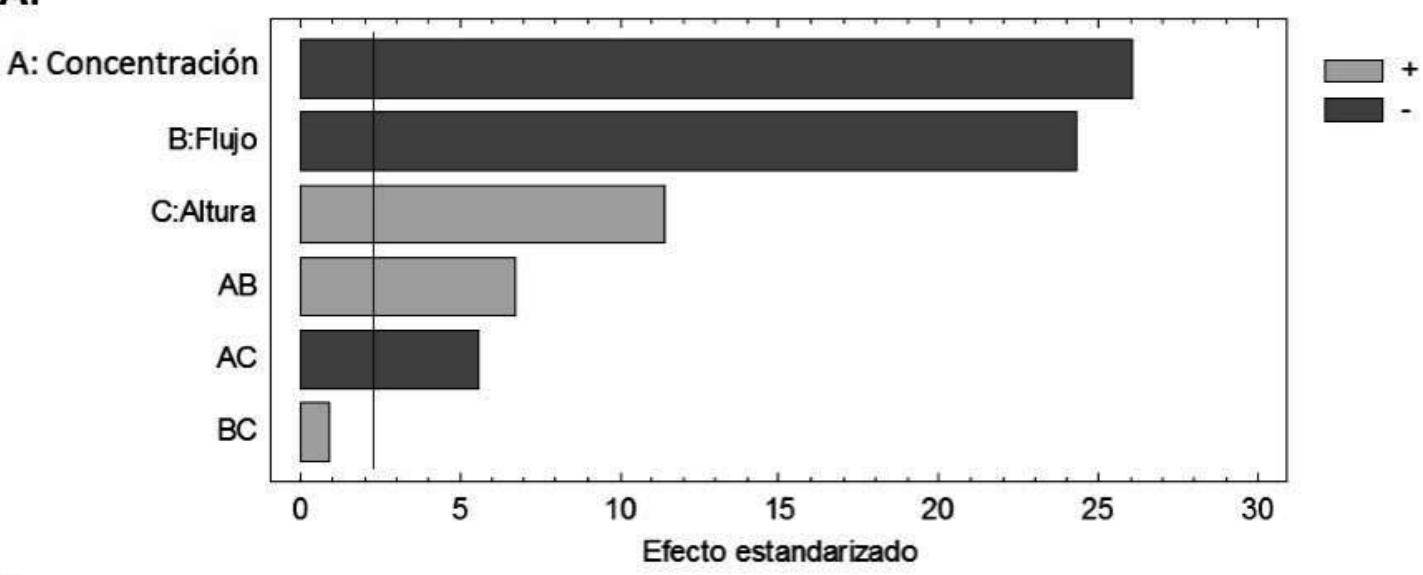

B.

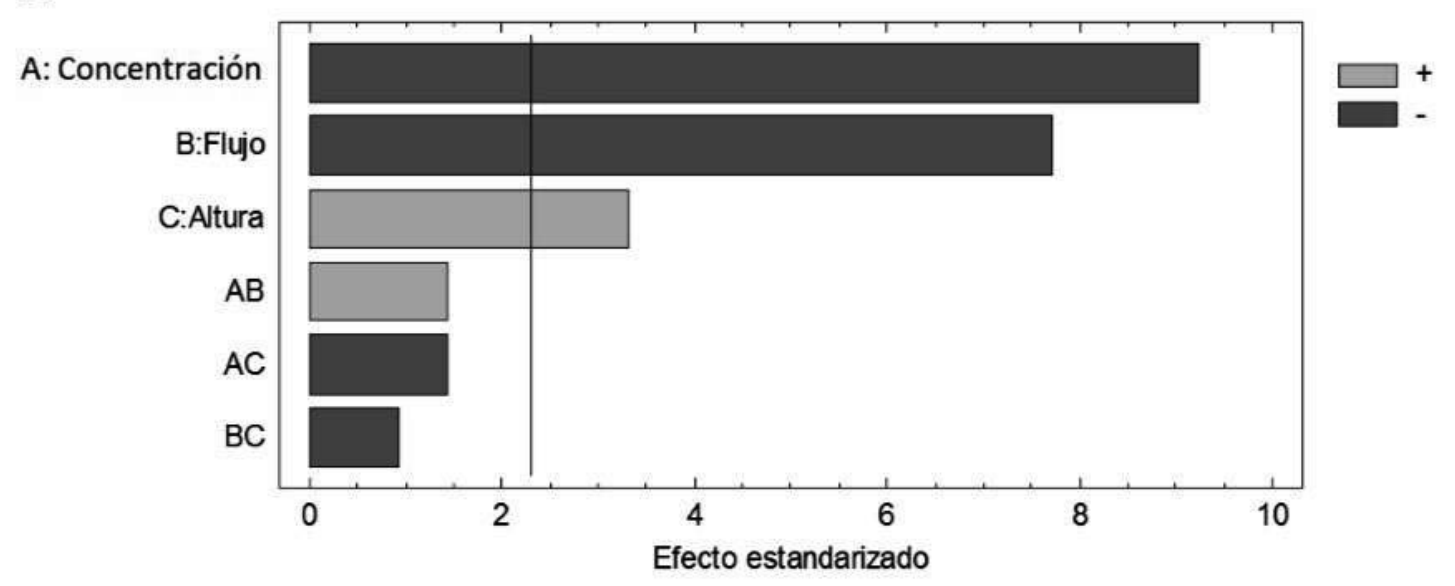

Figura 3. Diagrama de Pareto. A) borra de café y B) borra de café modificada.

ello, si se aumenta la concentración, disminuye la capacidad de adsorción de los adsorbentes y da como resultado, que un aumento de la concentración inicial trae como resultado tiempos de rupturas cortos. La misma situación se presenta para al flujo volumétrico, pues a flujos volumétricos bajos, aumenta el tiempo de ruptura y la capacidad de adsorción.

De esta investigación, se puede concluir que, tanto la borra de café sin tratar como modificada, a partir de su tratamiento con $\mathrm{H}_{3} \mathrm{PO}_{4}$ y su empleo como material adsorbente en la remoción del colorante DB2, en una columna de lecho fijo, tienen una química superficial diversa, con grupos funcionales orgánicos oxigenados, que le confieren características ácidas, importantes al momento de establecer interacciones adsorbente-adsorbato. Del estudio dinámico, las mejores condiciones de operación se alcanzaron a $6 \mathrm{~cm}$ de altura del lecho, flujo volumétrico de $2 \mathrm{~cm}^{3} \mathrm{~min}^{-1}$ y concentración de entrada de $8 \mathrm{mgdm}^{-3}$, con valores de capacidad máxima de adsorción de 0,33 y 0,40 $\mathrm{mgg}^{-1}$, para la borra de café sin tratar y modificada, respectivamente. De las curvas de ruptura obtenidas, se muestra que, tanto el tiempo de ruptura como la capacidad de adsorción, aumentan cuando se incrementa la altura del lecho y disminuyen el flujo volumétrico y la concentración inicial, siendo este último, el factor que más afecta la capacidad de la columna. Por último, los datos experimentales muestran un ajuste aceptable al modelo matemático BDST.

Agradecimientos: Los autores de este artículo agradecen la colaboración del Grupo Interdisciplinario de Investigación en Energía y Medio Ambiente de la Universidad Autónoma del Caribe y al Grupo de Investigación en Biotecnología de Microalgas, Fisicoquímica Aplicada y Estudios Ambientales de la Universidad del Atlántico. Conflicto de intereses: El manuscrito fue preparado y revisado con la participación de todos los autores, quienes declaramos que no existe ningún conflicto de intereses que ponga en riesgo la validez de los resultados presentados. 
Tabla 3. Análisis de varianza para la capacidad de adsorción de los adsorbentes.

\begin{tabular}{|c|c|c|c|c|c|}
\hline \multicolumn{6}{|c|}{ Borra de café } \\
\hline Fuente & Suma de cuadrados & Gl & Cuadrado medio & Razón-F & Valor-p \\
\hline \multicolumn{6}{|l|}{ EFECTOS PRINCIPALES } \\
\hline A: concentración & $4,95063 \times 10^{-4}$ & 1 & $4,95063 \times 10^{-4}$ & 678,94 & 0,0000 \\
\hline B: flujo & $4,30562 \times 10^{-4}$ & 1 & $4,30562 \times 10^{-4}$ & 590,49 & 0,0000 \\
\hline C: altura del lecho & $9,50625 \times 10^{-5}$ & 1 & $9,50625 \times 10^{-5}$ & 130,37 & 0,0000 \\
\hline \multicolumn{6}{|l|}{ INTERACCIONES } \\
\hline AB: Concentración x flujo & $3,30625 \times 10^{-5}$ & 1 & $3,30625 \times 10^{-5}$ & 45,34 & 0,0001 \\
\hline AC: Concentración $\mathrm{x}$ altura & $2,25625 \times 10^{-5}$ & 1 & $2,25625 \times 10^{-5}$ & 30,94 & 0,0004 \\
\hline BC: Flujo $\mathrm{x}$ altura & $5,62500 \times 10^{-7}$ & 1 & $5,62500 \times 10^{-7}$ & 0,77 & 0,4026 \\
\hline RESIDUOS & $6,56250 \times 10^{-6}$ & 9 & $7,29167 \times 10^{-7}$ & & \\
\hline TOTAL (CORREGIDO) & $1,08340 \times 10^{-3}$ & 15 & & & \\
\hline \multicolumn{6}{|c|}{ Borra de café modificada } \\
\hline Fuente & Suma de Cuadrados & Gl & Cuadrado Medio & Razón-F & Valor-p \\
\hline \multicolumn{6}{|l|}{ EFECTOS PRINCIPALES } \\
\hline A: concentración & $7,84 \times 10^{-4}$ & 1 & $7,84 \times 10^{-4}$ & 132,51 & 0,0000 \\
\hline B: flujo & $4,84 \times 10^{-4}$ & 1 & $4,84 \times 10^{-4}$ & 81,80 & 0,0000 \\
\hline C: altura del lecho & $1,10 \times 10^{-4}$ & 1 & $1,10 \times 10^{-4}$ & 18,63 & 0,0019 \\
\hline \multicolumn{6}{|l|}{ INTERACCIONES } \\
\hline AB: Concentración x flujo & $2,500 \times 10^{-5}$ & 1 & $2,500 \times 10^{-5}$ & 4,23 & 0,0700 \\
\hline AC: Concentración $\mathrm{x}$ altura & $1,225 \times 10^{-5}$ & 1 & $1,225 \times 10^{-5}$ & 2,07 & 0,1840 \\
\hline BC: Flujo $\mathrm{x}$ altura & $1,225 \times 10^{-5}$ & 1 & $1,225 \times 10^{-5}$ & 2,07 & 0,1840 \\
\hline RESIDUOS & $5,325 \times 10^{-5}$ & 9 & $5,916 \times 10^{-6}$ & & \\
\hline TOTAL (CORREGIDO) & $1,481 \times 10^{-3}$ & 15 & & & \\
\hline
\end{tabular}

\section{REFERENCIAS}

1. ADEGOKE,K.A.; BELLO, O.S. 2015. Dye sequestration using agricultural wastes as adsorbents. Water Resources and Industry 12(C):8-24

2. ALBIS, A.; MARTÍNEZ, J.; SANTIAGO, P. 2017. Remoción de zinc (II) de soluciones acuosas usando cáscara de yuca (Manihot esculenta): experimentos en columna. Prospectiva. (Colombia). 15(1):16-28.

3. BOEHM, H.P. 1966. Chemical identification of surface groups. Adv. Catal. (United States). 16:179-274.

4. BOHART, G.; ADAMS, E. 1920. Some aspects of the behavior of charcoal with respect to chlorine. J. Am. Chem. Soc. (United States). 42:523-544.
5. CASTELLAR, G.; ANGULO, E.; ZAMBRANO, A.; CHARRIS, D. 2013. Equilibrio de adsorción del colorante azul de metileno sobre carbón activado. Rev. U.D.C.A Act. \& Div. Cient. (Colombia). 16(1):263-271.

6. COLPAS, F.; TARÓN, A.; FONG, W. 2015. Analisis del desarrollo textural de carbones activados preparados a partir de zuro de maíz. Temas Agrarios. (Colombia). 20(1):103-112.

7. DEVI, R.; SING, V.; KUMAR, A. 2008. COD and BOD reduction from coffee processing wastewater using avocado peel carbon. Bioresour. Technol. 99:18531860. 
8. DIAS, S.; BISINELLA, F.; NIVALDO, A.; ESPINOZAQUIÑONES, F.; MARIN, P.; DIMITROV, A.; BORBA, C. 2017. Kinetic, equilibrium and thermodynamic phenomenological modeling of reactive dye adsorption onto polymeric Adsorbent. Chem. Eng. J. (Netherlands). 307:466-475.

9. EL MESSAOUDI, N.; EL KHOMRI, M.; DBIK, A.; BENTAHAR, S.; LACHERAI, A.; BAKIZ, B. 2016. Biosorption of Congo red in a fixed-bed column from aqueous solution using jujube shell: Experimental and mathematical modeling. J. Environ. Chem. Eng. (United Kingdom). 4(4):3848-3855.

10. ELSHERBINY, A. 2013. Adsorption kinetics and mechanism of acid dye onto montmorillonite from aqueous solutions: stopped-flow measurements. Appl. Clay Sci. (Netherlands). 83-84:56-62.

11. FERNÁNDEZ, M.E.; NUNELL, G.V.; BONELLI P.R.; CUKIERMAN, A.L. 2014. Activated carbon developed from orange peels: Batch and dynamic competitive adsorption of basic dyes. Ind. Crops Prod. (Netherlands). 62:437-445.

12. GUTIÉRREZ, A. 2002. Café, antioxidantes y protección a la salud. Medisan. (Cuba). 6(4):72-81.

13. R. HUTCHINS. 1973. New method simplifies design of activated carbon systems. Am. J. Chem. Eng. (United States). 80:133-138.

14. JUNG, K.; CHOI, B.; HWANG, M.; JEONG, T.; AHN, K. 2016. Fabrication of granular activated carbons derived from spent coffee grounds by entrapment in calcium alginate beads for adsorption of acid orange 7 and methylene blue. Bioresour. Technol. 219:185195.

15. KYZAS, G.Z.; LAZARIDIS, N.K.; MITROPOULOS, A. 2012. Removal of dyes from aqueous solutions with untreated coffee residues as potential low-cost adsorbents: Equilibrium, reuse and thermodynamic approach. Chem. Eng. J. 189-190:148-159.

16. LAFI, R.; HAFIANE, A. 2016. Removal of methyl orange (MO) from aqueous solution using cationic surfactants modified coffee waste (MCWs). J. Taiwan Inst. Chem. Eng. (United Kingdom). 58:424-433.

17. MA, X.; OUYANG, F. 2013. Adsorption properties of biomass-based activated carbon prepared with spent coffee grounds and pomelo skin by phosphoric acid activation. Appl. Surf. Sci. 268:566-570.
18. MYGLOVETS, M.; PODDUBNAYA, O.; SEVASTYANOVA, O.; LINDSTRO M.; GAWDZIK, B.; SOBIESIAK, M.; TSYBA, M.; SAPSAY, V.; KLYMCHUK, D.; PUZIY, A. 2014. Preparation of carbon adsorbents from lignosulfonate by phosphoric acid activation for the adsorption of metal ions. Carbon. (United Kingdom). 80:771-783.

19. MOHAMMED, N.; GRISHKEWICH, N.; AMBROSE, H.; BERRY, R.; CHIU, K. 2016. Continuous flow adsorption of methylene blue by cellulosenanocrystal-alginate hydrogel beads in fixed bed columns. Carbohydr Polym. (United Kingdom). 136:1194-1202.

20. NAZARI, G.; ABOLGHASEMI, H.; ESMAIELI, M.; SADEGHI POUYA, E. 2016. Aqueous phase adsorption of cephalexin by walnut shell-based activated carbon: A fixed-bed column study. Appl. Surf. Sci. 375:144-153.

21. NUNELL, G.V.; FERNÁNDEZ, M.E.; BONELLI, P.R.; CUKIERMAN, A.L. 2012. Conversion of biomass from an invasive species into activated carbons for removal of nitrate from wastewater. Biomass Bioenerg. (United Kingdom). 44:87-95.

22. PACHATHU, A.; PONNUSAMY, K.; VENKAT, S.K. 2016. Packed bed column studies on the removal of emulsified oil from water using raw and modified bagasse and corn husk. J. Mol. Liq. (Netherlands). 223:2561263.

23. PUERTA, G.I. 2011. Composición química de una taza de café. Avances Técnicos. (Colombia). 414:1-12.

24. TALAT, M.; MOHAN, S.; DIXITA, V.; KUMAR D.; HADI, S.; NATH O. 2018. Effective removal of fluoride from water by coconut husk activated carbon in fixed bed column: Experimental and breakthrough curves analysis. Groundwater Sustainable Developm. (Netherlands). 7:48-55.

25. SALEH, T.; AL-ABSI, A. 2017. Kinetics, isotherms and thermodynamic evaluation of amine functionalized magnetic carbon for methyl red removal from aqueous solutions. J. Mol. Liq. 248: 577-585.

26. TEHRANI, N.; AZNAR, J.; KIROS, Y. 2015. Coffee extract residue for production of ethanol and activated carbons. J. Clean. Prod. (Netherlands). 91:64-70.

27. SHANMUIGAM, D.; ALAGAPPAN, M.; RAJAN, R.K. 2016. Bench-scale packed bed sorption of Cibacron blue F3GA using lucrative algal biomass. AEJ. (Egypt). 55(3):2995-3003. 
28. URRIBARRÍ, A.; ZABALA, A.; SÁNCHEZ, J.; ARENAS, E.; CHANDLER, C.; RINCÓN, M.; GONZÁLEZ, E.; AIELLO, C. 2014. Evaluación del potencial de la borra de café como materia prima para la producción de biodiesel. Multiciencias. (Venezuela). 14(2):129-139.

29. VALENCIA, J.; CASTELLAR, G. 2013. Predicción de las curvas de ruptura para la remoción de plomo (II) en disolución acuosa sobre carbón activado en una columna empacada. Rev. Fac. Ing. Univ Ant. (Colombia). 66:141-158.

30. VILLADA, Y.; HORMAZA, A.; CASIS, N. 2014. Uso de la cascarilla de arroz para la remoción de azul de metileno en columnas de lecho empacado. Revista Tecno Lógicas (Colombia). 17(33):43-54.
31. YAKOUT, S.; SHARAF, G. 2016. Characterization of activated carbon prepared by phosphoric acid activation of olive stones. Arab. J. Chem. (Saudi Arabia). 9:1155-1162.

Recibido: Agosto 8 de 2017

Aceptado: Septiembre 3 de 2018

\section{Cómo citar:}

Castellar-Ortega, G.C.; Cely-Bautista, M.M.; Cardozo-Arrieta, B.M.; Angulo-Mercado, E.R.; Plaza-Vega, M.E. 2018. Adsorción del colorante azul directo 2 sobre borra de café en una columna de lecho fijo a escala de laboratorio. Rev. U.D.C.A Act. \& Div. Cient. 21(2): 531-541. https://doi.org/10.31910/rudca.v21.n2.2018.971 